\title{
Spontaneous EEG-Functional MRI in Mesial Temporal Lobe Epilepsy: Implications for the Neural Correlates of Consciousness
}

\author{
Zheng Wang, ${ }^{1}$ Loretta Norton, ${ }^{2}$ R. Matthew Hutchison, ${ }^{3}$ \\ John R. Ives, ${ }^{4}$ and Seyed M. Mirsattari ${ }^{2,4,5}$ \\ ${ }^{1}$ Department of Psychology, Vanderbilt University, Nashville, 37211 TN, USA \\ ${ }^{2}$ Department of Psychology, University of Western Ontario, London, ON, Canada N6A 5A5 \\ ${ }^{3}$ Department of Physiology and Pharmacology, University of Western Ontario, London, ON, Canada N6A 5A5 \\ ${ }^{4}$ Department of Clinical Neurological Sciences, University of Western Ontario, London, ON, Canada N6A 5A5 \\ ${ }^{5}$ Department of Medical Biophysics, University of Western Ontario, London, ON, Canada N6A 5A5
}

Correspondence should be addressed to Seyed M. Mirsattari, smirsat2@uwo.ca

Received 20 September 2011; Revised 21 November 2011; Accepted 19 December 2011

Academic Editor: Warren T. Blume

Copyright ( 2012 Zheng Wang et al. This is an open access article distributed under the Creative Commons Attribution License, which permits unrestricted use, distribution, and reproduction in any medium, provided the original work is properly cited.

\begin{abstract}
The combination of electroencephalography (EEG) and functional magnetic resonance imaging (fMRI) has been shown to have great potential for providing a greater understanding of normal and diseased states in both human and animal studies. Simultaneous EEG-fMRI is particularly well suited for the study of epilepsy in that it may reveal the neurobiology of ictal and interictal epileptiform discharges and noninvasively localize epileptogenic foci. Spontaneous, coherent fluctuations of neuronal activity and the coupled hemodynamic responses have also been shown to provide diagnostic markers of disease, extending our understanding of intrinsically structured ongoing brain activity. Following a short summary of the hardware and software development of simultaneous EEG-fMRI, this paper reviews a unified framework of integrating neuronal and hemodynamic processes during epileptic seizures and discusses the role and impact of spontaneous activity in the mesial temporal lobe epilepsies with particular emphasis on the neural and physiological correlates of consciousness.
\end{abstract}

\section{Introduction}

Temporal lobe epilepsy (TLE) manifests with partial seizures whose semiology can reflect a dynamic interplay among several anatomical divisions of the temporal lobe, in addition to their sites of origin [1]. Approximately $20 \%-30 \%$ of TLE patients are intractable to treatment with antiepileptic drugs though they may still benefit from surgical resection of the epileptogenic focus (EF) [2]. It is, therefore, essential to localize the EF in patients with focal epilepsy and to discern the large-scale cortical and subcortical networks involved in seizure generation. This is best achieved with the use of simultaneous, multimodal techniques that are able to elucidate complex functional relationships through converging or parallel operations. The development of electroencephalogram (EEG) recording of epileptic patients inside a magnetic resonance imaging (MRI) scanner was largely driven by the necessity of localizing and delineating mesial or deeply originating EF electrically and metabolically during presurgical evaluation [3-7]. The concurrent measures allow for the monitoring of abnormal hypersynchronous events in the EF, as well as the full-brain coverage of variations in blood flow and oxygenation in response to epileptic discharges conducive to a wide range of research applications within epileptology. Simultaneous EEG-fMRI has the potential to become a routine diagnostic test, complementing the diagnostic accuracy of clinical acumen and EEG to greatly improve medical and surgical managements of patients with intractable epilepsy, a goal that was set since its first introduction [4].

Investigation of spontaneous, low-frequency, and bloodoxygenation-level-dependent (BOLD) fluctuations measured by fMRI has revealed spatially organized and distributed brain networks [8-16]. The coherent patterns of hemodynamic oscillations occur in the absence of any overt 
task and as such are referred to as resting-state networks (RSNs). RSNs are believed to be the epiphenomenon of endogenous neural activity [17] and shaped by structural connections [16, 18-21] though their functional significance and neuronal correlate remain an active area of research $[22,23]$. Alterations of RSNs have recently been reported in several conditions, including psychiatric and developmental disorders [24], Alzheimer's disease [25], schizophrenia [26], coma [14], and epilepsy [27-30], revealing changes in connectivity between network nodes. By contrast, spontaneous neuronal rhythm measured using EEG evince endogenous patterns of connectivity and synchronicity underlying states of attention, perception, and consciousness [31]. A comprehensive understanding of the functional network dynamics related to the electrical activity recorded from the EF during ictal and interictal epileptiform discharges (IEDs) remains elusive [32-35]. However, empirical evidence does indicate disruption of the normal distributed networks; temporal lobe IEDs of patients with complex partial seizures secondary to TLE have been reported to affect the activity in regions comprising the default-mode RSN (discussed below) [36]. Therefore, spontaneous neural activity and its associated hemodynamic manifestation (BOLD contrast) allow not only for the noninvasive exploration of temporal and spatial patterns of the brain but can also be envisaged as early diagnostic or prognostic markers of disease states [14, 24, 3743]. This review places an emphasis on the fundamentals of spontaneous EEG-fMRI and extends the unified framework we previously proposed to a new avenue of exploring the neuronal and physiological basis underlying intrinsic brain activity. Only studies relevant to specific aspects of simultaneous EEG-fMRI in mesial TLE (mTLE) studies in the context of resting state including seizure-induced alterations of the conscious state are discussed in here.

\section{Emerging Issue in Simultaneous EEG-fMRI Acquisition}

The prevalent strategy is to continuously sample the interictal and ictal events while measuring the BOLD signal for spontaneous EEG-fMRI study. Unfortunately, continuousrecording EEG on epileptic patients inside the magnet suffers various kinds of noise problems such as muscle contraction motions, eye movements, perspiration, $50 / 60 \mathrm{~Hz}$ power interference, and ballistocardiogram [44]. Motion-related artifacts of patients such as involuntary gross head movements, swallowing, and coughing can also impose adverse consequence on both EEG signals and MRI images. As such, these confounds make data acquisition in the high-field environment more difficult through deteriorating the shimming performance and causing unexpected susceptibility issues arising from magnetic field inhomogeneities at air-tissue interfaces. There have been a number of postprocessing methods developed to handle the epileptic electrophysiological data by focusing on the focal spike density [45], phase coherence [32-35, 46], and noise separation [47]. But, many of these artifacts including signal loss, image distortion, and poor BOLD contrast-to-noise ratio can occur both focally and globally in an unpredictable manner. They may also vary slowly with physiological vitals, extending beyond straightforward image misregistration and invoking more advanced mathematical techniques to mitigate these confounds. Independent component analysis (ICA) has emerged as a popular data-driven method that has been used successfully to remove ballistocardiogram and other MRI-related artifacts during the past decade [47-51]. For the purposes of reliably detecting clean, discrete interictal and ictal spike events, noise components either from statistically independent decomposition or adequately sampled recording in a specific reference channel can be simply subtracted out from an unsaturated raw EEG trace [6], or in more elaborate techniques such as adaptive noise cancellation [44]. Nevertheless, ICA has been successfully utilized to reveal intrinsic functional connectivity patterns of RSNs in human and animal fMRI data sets $[8,11,12,52]$.

Besides the difficulties summarized above, the relation between the detectable anatomical abnormalities, epileptic neuronal activity (i.e., various types of epileptic seizures), and functional MRI signal remains largely unclear $[53,54]$. This elusive relationship also generates a strong motivation for those seeking to improve the recording EEG inside the MRI scanner. One of many emerging issues is to detect the high-frequency oscillations with clinical instruments.

Previously BOLD changes have been reported to occur before the happening of IEDs in patients with focal epilepsies, suggesting the alteration of synchronized neuronal activity in the spike field before the generation of EEG spike [55]. Similar findings have been reported before the onset of epileptic seizures $[56,57]$. In the study by Jacobs and colleagues [55], the early focal BOLD changes were localized in the mesial temporal lobe where neuronal activity might not be detectable by the scalp EEG. Scalp potentials arise from the spatial summation of synchronous dipole moments over the neocortical volume with a weighting that depends upon the anisotropic conductivity of the cranium [58, 59]. Areas that are too deep or lack a sufficient amount of synchrony are unable to produce a measurable potential difference on the scalp. The "blurring" and low-pass filtering effects of the skull and scalp can be overcome with invasive depth recordings, as only a fraction of spikes recorded intracranially in mTLE patients are detectable by the EEG [59].

More recently with small clinical contacts and clinical amplifiers, high-frequency oscillations (HFOs, referred to as ripples $80-250 \mathrm{~Hz}$ and fast ripples $250-500 \mathrm{~Hz}$ ) have been recorded in animal and human subjects [60-62], suggesting a possible relation with ictogenesis $[61,63,64]$ and epileptogenesis [65]. Moreover, Châtillon and colleagues demonstrated that the contact size did not significantly affect HFO detection in intracerebral EEG recordings in a rat epilepsy model [60]. They have shown that the optimal size of a recording electrode should be dependent on the potential generation, distribution, amplitude, and frequency of the targeted signal, even though it was conventionally deemed that the contact size may influence $\mathrm{HFO}$ recording ability by affecting impedance and sampling volume. Specifically, they suggested there should be no difference in HFO detection in human recordings using contact from $0.036 \mathrm{~mm}^{2}$ to $1.698 \mathrm{~mm}^{2}$. The generation of the HFO varies in anatomical 
structures and pathological conditions that could lead to the difference in its detectability reported by different groups. Further research with regard to synchrony in large neuronal circuitry is needed for detailed and complete understanding of the underlying mechanism from a global network perspective. Note that analysis of changes in phase synchronization, frequency bands, and rhythmicity was not conducted by Jacobs and colleagues and presents an exciting avenue for future study.

\section{Correspondence between Spontaneous EEG and fMRI}

3.1. Methodological Consideration. The challenges of concurrent EEG-fMRI are not solely limited to noise removal, but also arise when developing the framework of analysis. This represents a fundamental issue which can influence the seeking of the correspondence between the measured spontaneous EEG and resting-state fMRI signals (see Figure 1 for a unified framework). Since little is known about the BOLD response to epileptic discharges prior to the analysis, two fundamental assumptions must be made to establish the link between neural activity and hemodynamic modulations. The first is that the shape and time course of the hemodynamic response function (HRF) derived from the normal human or animal subjects is also appropriate for epileptic brains $[3,27$, $66]$. The second is that the nature of neural hemodynamic coupling derived from an epileptic subject is homogenous across different brain regions regardless of the location inside or outside the epileptogenic focus. Judicious selection of an HRF and experimentally based assumptions about the nature of the cortical electrical activity are important first steps in the general linear model analysis of fMRI data sets [27, 49, 50]. Many of the published epilepsy studies using fMRI have been bound by these two underlying assumptions.

A growing body of evidence is challenging the validity of these two working hypotheses and suggest that the HRF and its uniformity of epilepsy patients deviate from the normal population $[55,66,68-70]$. The result has been a greater preference for the utilization of data-driven or exploratory methods such as ICA and temporal clustering analysis [71, 72]. A major advantage of data-driven analyses is the flexibility to circumvent a priori hypothesized HRF while simultaneously deducing the activated fMRI response [73]. However, it suffers an inherent deficit: the lack of statistical measure to assess the intended hypothesis. To be considered clinically useful, the epileptogenic zones defined by hemodynamic and metabolic modulation through ICA analysis must be spatially consistent and statistically robust across individuals and scanning sessions. Consequently, one has to rely on comparison of the results with the linear model method for validation $[49,50]$. McKeown has proposed a hybrid method which can gracefully navigate from a fully data-driven approach to a fully model-driven approach [74]. Building on this early work, we further improved it by replacing a vital priori hypothesis (presumed neuronal response) used in McKeown's work with the actual EEG signal and obtained statistically robust BOLD activation in an animal model of focal epilepsy [71]. With regard to the framework of EEGfMRI, the integration of superior spatial information provided by MR images and temporal information of EEG can be improved by linking them with hemodynamic transfer functions as we previously proposed. In our framework, the variation of the HRF can be estimated rather than simply assuming a fixed canonical response. Another advantage is that intermodal, interregional, and interindividual variability can be explicitly taken into account in the estimation. Thus, it becomes more straightforward to fit epileptic discharge events into continuous fMRI recordings under a spontaneous condition since this type of data does not contain sparse/blocked stimulus inputs [41].

3.2. Neuronal and Physiological Consideration. Paradoxical imaging changes can occur in the nonepileptogenic and epileptogenic zones that do not always accurately reflect the underlying electrical activity $[57,66]$. During absence seizures, it still remains largely unknown if a decrease in fMRI signal during seizure activity has been found in those regions such as spike-wave discharges, relative silence, or some other electrophysiological phenomenon occurring [27, 49, 75-79]. From a large-scale network perspective, it is uncertain if in the complex partial seizures of temporal lobe origin that either reduced excitation or increased inhibition (or both) results in decreased activity in the frontoparietal cortex [8082].

The characterization of the neuronal correlate of spontaneous BOLD signal still remains an open issue. Typical observations of spontaneous EEG contain a complex spectral composition including the alpha rhythm $(8-12 \mathrm{~Hz}$ oscillations), sleep spindles ( $\sim 12-14 \mathrm{~Hz}$ oscillations), and individual IEDs [83]. BOLD fMRI contrast should be sensitive to these and any changes in neuronal function that result in alteration (either increase or decrease) of brain metabolism [84-86]. It should be noted that failure to consider the background EEG oscillations when seeking the electrical basis of the BOLD response may cause poor correlation between these two measures [69] and confusion when attempting to explain their direct correspondence [55, 87]. For example, evidence from simultaneous fMRI and depth recordings in monkeys and individuals undergoing invasive clinical monitoring has suggested that spiking, multiunit activity, and band-limited power changes in the gamma frequency range were the primary correlate of resting state fMRI activity and connectivity $[17,88]$. This is in conflict with other work in humans suggesting a direct linkage of low-frequency BOLD fluctuations and the posterior alpha rhythm [89]. Further, He and Raichle proposed that the low-frequency end of field potentials $(<4 \mathrm{~Hz}$, also termed as "slow cortical potential", SCP) is also correlated with BOLD signal in its raw spontaneous fluctuations in the light of its temporal scale overlapping with that of fMRI signal $[15,90]$. Correlation at or below delta band LFPs is also in agreement with a rat investigation [91]. De Munck and colleagues have further reported that power fluctuations of different EEG bands are significantly correlated and are similar to the alpha harmonics [89]. In striking contrast, other studies yield different results as to the role what other 


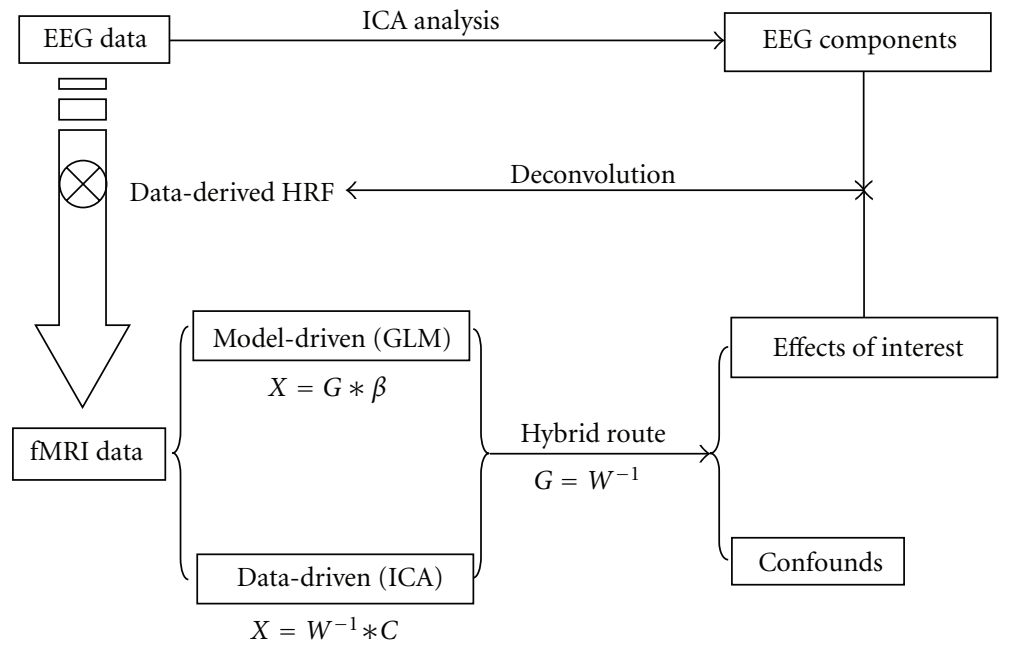

FIGURE 1: Unified framework of simultaneous EEG-fMRI analysis.

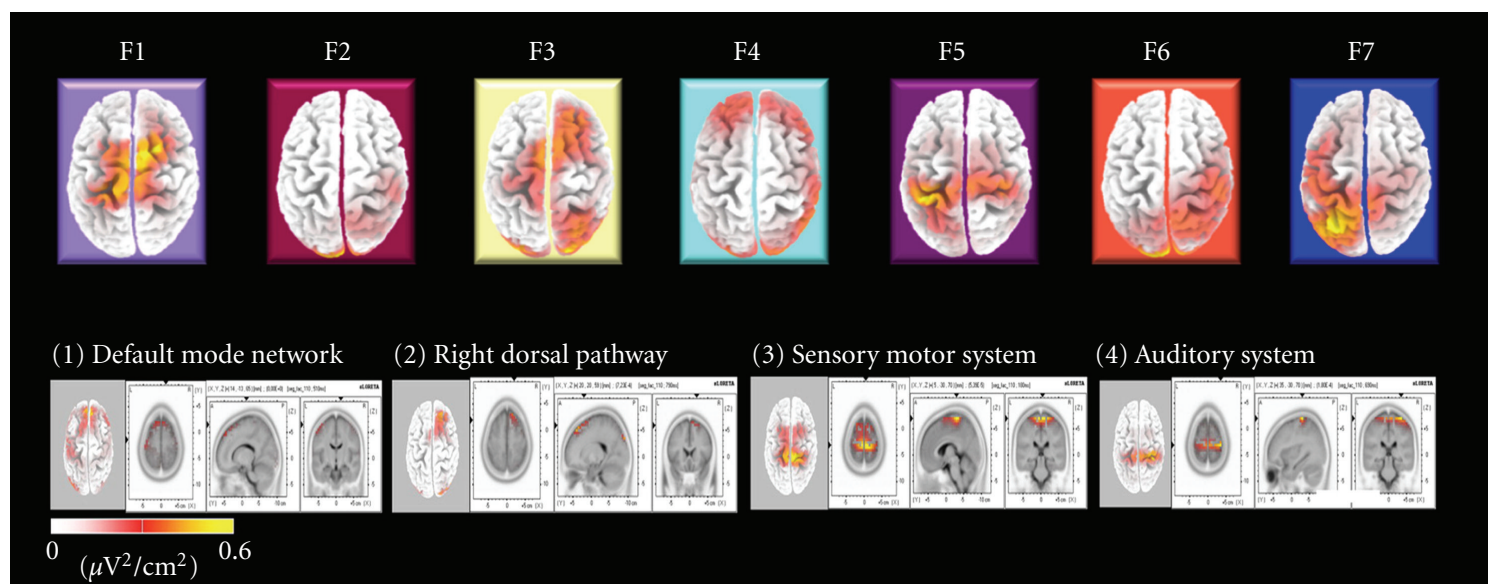

FIGURE 2: Statistical factor analysis on the microstates inferred by EEG and fMRI. The top row shows the sLORETA CSD maps $\left(\mu\right.$ V2/cm $\left.{ }^{2}\right)$ of the specific electrophysiological landscape or microstate, which is used to inform the fMRI analysis in four selected single subject at rest (the bottom row). In each column of the bottom row, on the left, a CSD map projected onto a 3D Talairach model of the brain while on the right axial, sagittal and coronal views of a microstate CSD map is shown. Reproduced and modified with permission from Musso et al. [67].

frequency bands play $[92,93]$, some suggesting unique frequency profiles across multiple bands representing characteristic EEG microstates $[67,94]$ (see Figure 2 for the EEG microstates informed fMRI analysis). It has yet to be adequately clarified how changes in distinct bands of electrical signal are related to the hemodynamic response under different states of brain activity.

Therefore, two important caveats should be noted when one attempts to resort to a consistent interpretation of these controversial observations. One is that intrinsic synchrony of brain activity is network specific and exists at multiple spatial levels [24]. Another is that spontaneous activity not only reflects the functional architecture of the brain by forming structured spatiotemporal profiles but can also encode traces of previous behavior history (memory retrieval) and predict future decisions in view of its "ongoing" internal representations [15]. In other words, the role of the brain oscillations within an interested frequency range could be highly contextual. Taken together, further study of humans and animal models with the use of simultaneous, spontaneous EEGfMRI recording is necessary to elucidate the complex, dynamic, behavior of intrinsic hemodynamic oscillations, and their neuronal correlate. Revealing the underlying mechanisms of brain oscillations, coupling, and functional significance will provide a valuable measure for the assessment of both normal and pathological brain functions, especially epilepsy.

\section{Alterations of Spontaneous EEG-fMRI in Mesial TLE}

TLE is often considered the prototype of localization-related epilepsy, even though evidence indicates a multiplicity of sources involving cortical and subcortical structures outside 

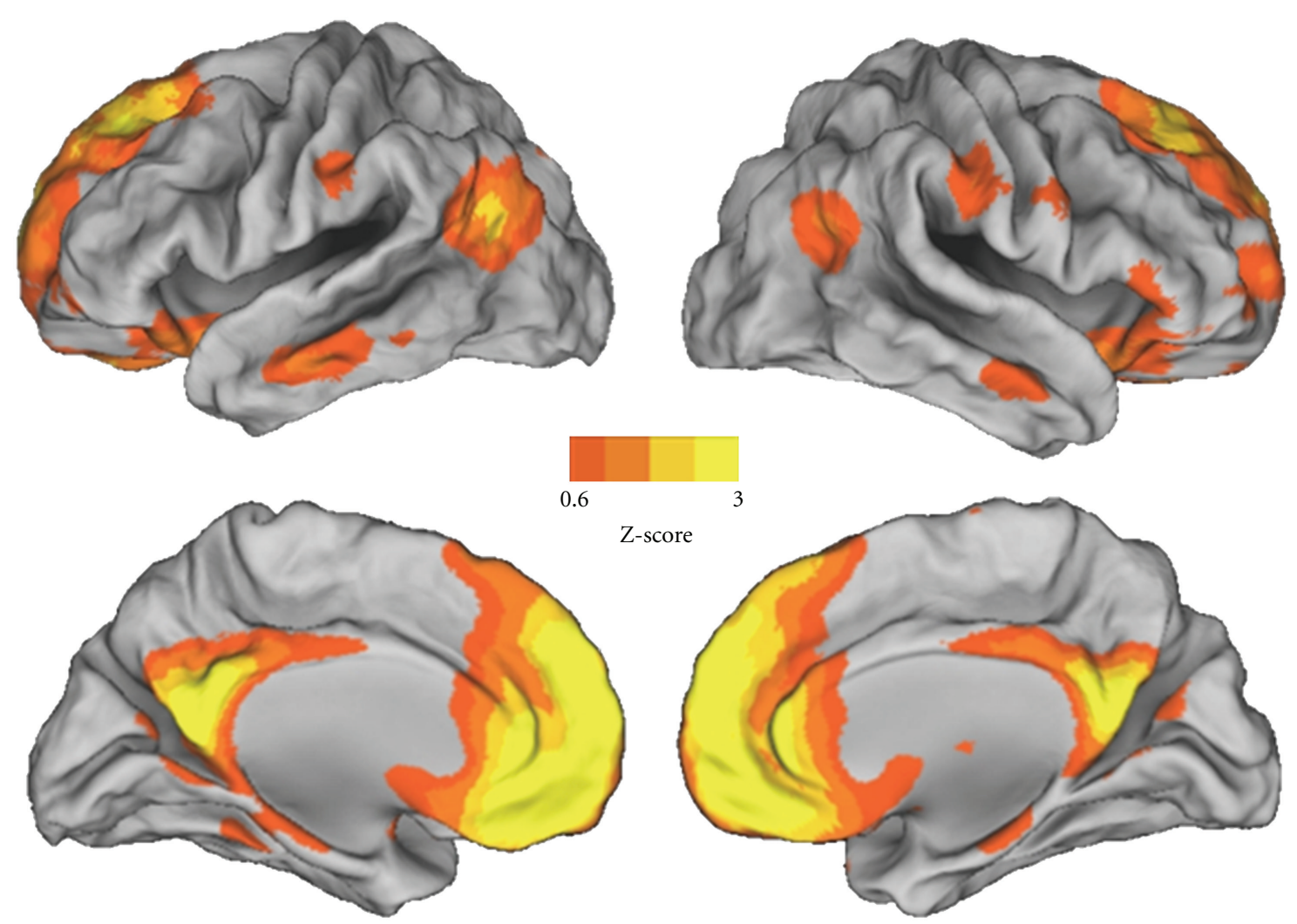

FIGURE 3: The human default-mode network represents a critical junction in the study of temporal lobe epilepsy, consciousness, and spontaneous brain activity. The resting-state network is displayed on a $3 \mathrm{D}$ cortical representation of the human brain. Overlaid color maps represent thresholded $\mathrm{z}$ scores derived using independent component analysis network of resting-state fMRI data $(N=13)$.

the epileptogenic temporal lobe [28]. For instance, mTLE is the most common type of focal epilepsy in adult patients and is usually caused by hippocampal sclerosis (HS) [29]. A large number of concurrent EEG-fMRI studies have sought fMRI activations during IEDs to localize the epileptogenic focus in their presurgical evaluation of patients with medically refractory partial epilepsies [54]. Surgical outcomes in mTLE are good, but far from optimal, particularly in long-term followup studies [41, 95]. One of potential reasons of poor clinical outcome could be that hemodynamic activation and deactivation patterns are not always of localizing merit since current understanding of neurovascular regulation is limited [53]. When studying functional connectivity of the mesial temporal lobe, one must consider that the subregions within temporal lobe structures are critical components of other cortical networks [35]. TLE focal seizure onset can propagate to subcortical structures impairing the ascending reticular activating system (ARAS) which mediates arousal and in turn deactivating the cortical hierarchy, particularly the large frontal-parietal associative cortical areas [96]. This fits within the "network inhibition hypothesis" suggesting that partial complex seizures that lead to impaired consciousness affect an entire network as opposed to only the local epileptogenic focus $[1,96,97]$.

Interestingly, these cortical areas are implicated in the default-mode network (DMN), a RSN, which comprises the posterior cingulate cortex/precuneus, lateral parietal cortex, ventral anterior cingulate cortex/mesial prefrontal cortex, angular gyrus, and inferior temporal cortex, in addition to the mesial temporal lobes (see Figure 3). Mesial TLE patients show significantly increased connectivity within the mesial temporal lobes and decreased connectivity within/between the frontal and parietal lobes implicated in the DMN [28]. In addition, cerebral blood flow (CBF) has been shown to increase in the medial thalamus correlating with a reduction in activity within the DMN regions [81]. Morgan et al. [72] showed increased negative connectivity across thalamic, brainstem, frontal, and parietal brain regions, in accordance with the idea that there is inhibited function in subcortical and cortical structures during ictal propagation. These findings suggest that both thalamocortical activation and suspension or disturbance of the default state contribute to the abnormality in responsiveness of patients with medial temporal origin. They are in concordance with what Gotman and coworkers found using concurrent EEG-fMRI. The brain could temporarily suspend baseline processes and engender deactivations in this higher-order associative network [27]. The overlap of cortical between the DMN and TLE may provide some context as to why altered consciousness occurs in TLE (see Figure 4 for illustration of the network inhibition hypothesis). 


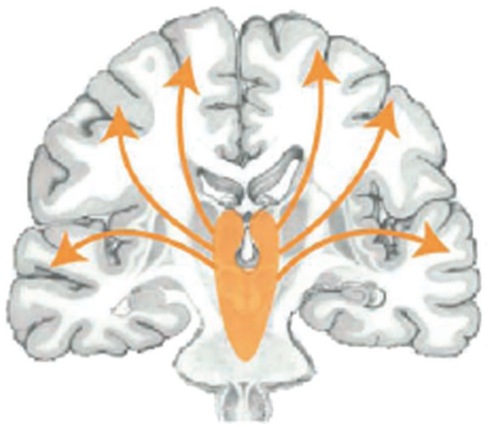

(a)

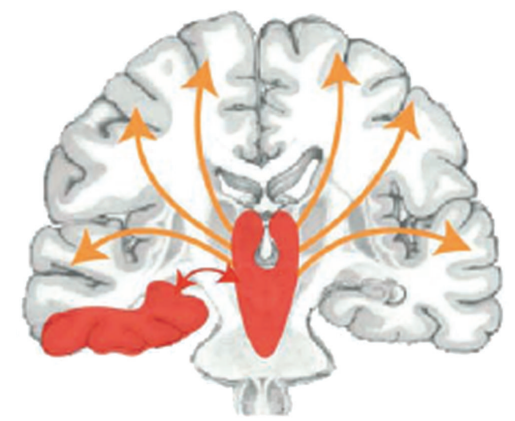

(c)

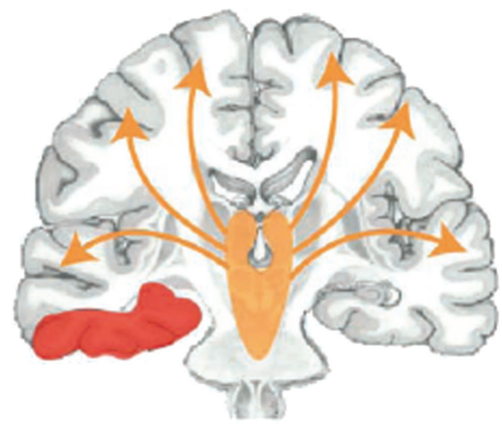

(b)

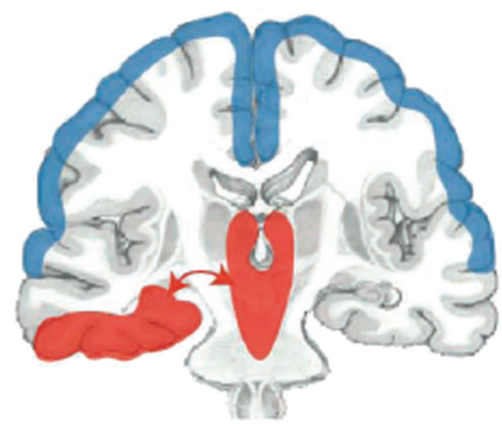

(d)

FIGURE 4: Network inhibition hypothesis for loss of consciousness during the onset and propagation of unilateral focal seizure in mesial temporal lobe. Reproduced with permission from Blumenfeld and Taylor [1].

The role of the DMN is still largely unclear, however, two hypotheses have been proposed relating to conscious awareness (for review see [98]). The DMN has been suggested to be responsible for unconstrained conscious internal mental processes such as mind wandering [99] or alternatively may involve low-level monitoring of the external environment $[10,100]$. Regardless of the fact that one or both hypotheses may be correct, both relate to awareness (either of self or environment), and thus the contents of consciousness. The potential role of the DMN governing conscious awareness has been investigated through imaging in altered states of consciousness where awareness is thought to be absent including such conditions as anesthesia [16, 101], sleep [102], vegetative state $[38,42,103]$, and coma $[14,37]$. Under light sedation in healthy subjects, Greicius et al. [101] found significantly reduced connectivity in the posterior cingulate cortex (PCC) within the DMN suggesting that focal reductions of the PCC may reflect decreased levels of consciousness. In deep sleep, a more natural occurrence of decreased consciousness, a decoupling of the medial prefrontal cortex to the rest of the cortical regions in DMN, have been shown to occur [102]. A disruption of functional connectivity in the DMN in vegetative state (VS), a pathological disorder of consciousness whereby a patient lacks awareness while regaining arousal, has also been reported. In three VS patients, it was found that the degree of functional connectivity within the network correlated with the severity of the neurological impairment [103]. An absence of DMN has also been reported in a VS patient who was scanned 21 years after anoxic injury
[42]. Vanhaudenhuyse et al. [43] examined DMN connectivity in 14 patients with range of altered states of consciousness including locked-in syndrome, minimally conscious, vegetative states, and comatose patients and found that the DMN connectivity decreased in proportion to the degree of consciousness impairment. When taken together, the integrity of the DMN may correlate with levels of consciousness, and a fully intact DMN may be needed for normal levels of consciousness to occur.

The DMN has also been proposed to have some other intrinsic role in functional brain organization [16]. Preserved DMN connectivity has been documented in one case of VS [38], in coma [14,37], and in a possible homologous network under isoflurane anesthesia in the monkey [16]. It has been suggested that DMN may be needed for consciousness to occur but cannot be exclusively responsible for conscious awareness [16, 37]. Possibly, DMN connectivity may be an indicator of the extent of cortical disruption and predict reversible impairments in consciousness [14].

The role of DMN in mediating consciousness is supported by known metabolic impairments in VS patients which occur in cortical areas known to be implicated in the DMN such as the prefrontal, temporoparietal association areas, and posterior cingulate cortex/precuneus [104]. In addition, the emergence from reversible vegetative state coincides with recovery-related metabolic changes in areas such as the precuneus and thalamus $[40,105]$. The DMN is an attractive candidate for the neural correlate of consciousness as it has a great deal of functional and structural connectivity 
across large associative cortical areas $[98,106]$ including long-range corticothalamic connections, in addition to its role in internal mental processes and/or involvement in gathering information about the external environment, as well as disruptions in altered states of consciousness. The DMN fits within the global workspace (GW) theory of consciousness that suggests long-distance connectivity between multiple cortical networks that usually operate separately work together in an organized fashion to enable consciousness to occur [107]. Intracerebral EEG signals in TLE seizures resulting in alterations in consciousness have been shown to cause oversynchronization in long-range corticocortical and corticothalamic connections [108]. Excessive synchrony of these connections, reported to be important in the GW, is thought to prevent the variation and complexity needed to allow for a conscious state [109]. Intracerebral EEG recordings in the thalamus and temporal lobe structures (hippocampus, entorhinal cortex, and neocortex), have shown increased neuronal synchrony with the occurrence of seizures and early loss of consciousness [35].

The concept of consciousness has long been central to epileptology since generalized seizures and complex partial seizures both can induce a variable degree of impairment in consciousness [31], as frequently observed in MTLE patients. There is a series of discussion on this theme most recently [109-115]. Besides linking the SCP to the resting BOLD fluctuations as discussed above, He and Raichle made a remarkable stride forward in arguing that this slow frequency of cortical oscillation might contribute directly to the emergence of consciousness [90]. Their work has triggered a debate in the field of whether the SCP per se in widespread cortical networks carries specific details sufficient to express our vivid daily conscious experience, as opposed to the proposed role of gamma oscillation established in higher level of cognitive experience like attention [116]. It has been well known that the contents of conscious states as well as the level of awareness are affected to varying degrees during different types of epileptic seizures [27, 31, 110, 111, 115]. Accordingly, epilepsy can be used to provide an easily accessible entry into the working mechanism of altered conscious states. In principle, carefully constructed experiments that manipulate the onset/offset and subjective level of consciousness loss could dissociate hardly defined awareness from activity of either the SCP or gamma oscillation (or both).

\section{Conclusions}

Over the past decade, simultaneous EEG-fMRI recording has become technically feasible with applications in multiple areas of basic and applied sciences. Converging evidence from fMRI and depth EEG in humans and animals has already revealed that generalized seizures do not affect all brain areas indiscriminately, whereas complex partial seizures alter functional brain activity less focally than previously thought. Spontaneous EEG-fMRI investigations into topics such as asymmetry of the background activity in the homeostatic neural networks, paroxysmal focal delta or theta oscillations, and other nonepileptic but abnormal phenomena may provide additional clinically meaningful information, particularly relevant to impairment of conscious experience in patients. It will greatly enhance our understanding of the underlying mechanism in generation of generalized seizures, provide unique insight into the brain regions involved in the generation and/or propagation of epileptiform activity, determine the neural substrate of various forms of dyscognitive seizures, and offer clinicians neuronally validated spatial guidance for surgical planning to benefit patients with medically intractable TLE.

\section{Acknowledgment}

This work was partly funded by The Physicians' Services Incorporated Foundation (SM).

\section{References}

[1] H. Blumenfeld and J. Taylor, "Why do seizures cause loss of consciousness?” Neuroscientist, vol. 9, no. 5, pp. 301-310, 2003.

[2] S. Wiebe, W. T. Blume, J. P. Girvin, and M. Eliasziw, "A randomized, controlled trial of surgery for temporal-lobe epilepsy," New England Journal of Medicine, vol. 345, no. 5, pp. 311-318, 2001.

[3] R. I. Goldman, J. M. Stern, J. Engel Jr., and M. S. Cohen, "Acquiring simultaneous EEG and functional MRI," Clinical Neurophysiology, vol. 111, no. 11, pp. 1974-1980, 2000.

[4] J. R. Ives, S. Warach, F. Schmitt, R. R. Edelman, and D. L. Schomer, "Monitoring the patient's EEG during echo planar MRI," Electroencephalography and Clinical Neurophysiology, vol. 87, no. 6, pp. 417-420, 1993.

[5] L. Lemieux, P. J. Allen, F. Franconi, M. R. Symms, and D. R. Fish, "Recording of EEG during fMRI experiments: patient safety," Magnetic Resonance in Medicine, vol. 38, no. 6, pp. 943-952, 1997.

[6] S. M. Mirsattari, J. R. Ives, F. Bihari, L. S. Leung, R. S. Menon, and R. Bartha, "Real-time display of artifact-free electroen -cephalography during functional magnetic resonance imaging and magnetic resonance spectroscopy in an animal model of epilepsy," Magnetic Resonance in Medicine, vol. 53, no. 2, pp. 456-464, 2005.

[7] S. Warach, J. R. Ives, G. Schlaug et al., "EEG-triggered echoplanar functional MRI in epilepsy," Neurology, vol. 47, no. 1, pp. 89-93, 1996.

[8] C. F. Beckmann, M. DeLuca, J. T. Devlin, and S. M. Smith, "Investigations into resting-state connectivity using independent component analysis," Philosophical Transactions of the Royal Society B, vol. 360, no. 1457, pp. 1001-1013, 2005.

[9] B. Biswal, F. Z. Yetkin, V. M. Haughton, and J. S. Hyde, "Functional connectivity in the motor cortex of resting human brain using echo-planar MRI," Magnetic Resonance in Medicine, vol. 34, no. 4, pp. 537-541, 1995.

[10] D. A. Gusnard and M. E. Raichle, "Searching for a baseline: functional imaging and the resting human brain," Nature Reviews Neuroscience, vol. 2, no. 10, pp. 685-694, 2001.

[11] R. M. Hutchison, S. M. Mirsattari, C. K. Jones, J. S. Gati, and L. S. Leung, "Functional networks in the anesthetized rat brain revealed by independent component analysis of resting-state FMRI," Journal of Neurophysiology, vol. 103, no. 6, pp. 3398-3406, 2010. 
[12] R. M. Hutchison, L. S. Leung, S. M. Mirsattari, J. S. Gati, R. S. Menon, and S. Everling, "Resting-state networks in the macaque at 7T," NeuroImage, vol. 56, no. 3, pp. 1546-1555, 2011.

[13] R. M. Hutchison, T. Womelsdorf, J. S. Gati et al., "Restingstate connectivity identifies distinct functional networks in macaque cingulate cortex," Cerebral Cortex. In press.

[14] L. Norton, R. M. Hutchison, D. L. Lee et al., "Disruptions of functional connectivity in the default mode network of comatose patients," Neurology, vol. 78, no. 3, pp. 175-181, 2012.

[15] M. E. Raichle, “Two views of brain function," Trends in Cognitive Sciences, vol. 14, no. 4, pp. 180-190, 2010.

[16] J. L. Vincent, G. H. Patel, M. D. Fox et al., "Intrinsic functional architecture in the anaesthetized monkey brain," $\mathrm{Na}$ ture, vol. 447, no. 7140, pp. 83-86, 2007.

[17] A. Shmuel and D. A. Leopold, "Neuronal correlates of spontaneous fluctuations in fMRI signals in monkey visual cortex: implications for functional connectivity at rest," Human Brain Mapping, vol. 29, no. 7, pp. 751-761, 2008.

[18] M. D. Greicius, K. Supekar, V. Menon, and R. F. Dougherty, "Resting-state functional connectivity reflects structural connectivity in the default mode network," Cerebral Cortex, vol. 19, no. 1, pp. 72-78, 2009.

[19] C. J. Honey, O. Sporns, L. Cammoun et al., "Predicting human resting-state functional connectivity from structural connectivity," Proceedings of the National Academy of Sciences of the United States of America, vol. 106, no. 6, pp. 2035-2040, 2009.

[20] C. Kelly, L. Q. Uddin, Z. Shehzad et al., "Broca's region: linking human brain functional connectivity data and nonhuman primate tracing anatomy studies," European Journal of Neuroscience, vol. 32, no. 3, pp. 383-398, 2010.

[21] D. S. Margulies, J. L. Vincent, C. Kelly et al., "Precuneus shares intrinsic functional architecture in humans and monkeys," Proceedings of the National Academy of Sciences of the United States of America, vol. 106, no. 47, pp. 20069-20074, 2009.

[22] R. L. Buckner and J. L. Vincent, "Unrest at rest: default activity and spontaneous network correlations," NeuroImage, vol. 37, no. 4, pp. 1091-1096, 2007.

[23] M. D. Fox and M. E. Raichle, "Spontaneous fluctuations in brain activity observed with functional magnetic resonance imaging," Nature Reviews Neuroscience, vol. 8, no. 9, pp. 700711, 2007.

[24] D. Zhang and M. E. Raichle, "Disease and the brain's dark energy," Nature Reviews Neurology, vol. 6, no. 1, pp. 15-28, 2010.

[25] M. D. Greicius, G. Srivastava, A. L. Reiss, and V. Menon, "Default-mode network activity distinguishes Alzheimer's disease from healthy aging: evidence from functional MRI," Proceedings of the National Academy of Sciences of the United States of America, vol. 101, no. 13, pp. 4637-4642, 2004.

[26] S. Whitfield-Gabrieli, H. W. Thermenos, S. Milanovic et al., "Hyperactivity and hyperconnectivity of the default network in schizophrenia and in first-degree relatives of persons with schizophrenia," Proceedings of the National Academy of Sciences of the United States of America, vol. 106, no. 4, pp. 1279-1284, 2009.

[27] J. Gotman, C. Grova, A. Bagshaw, E. Kobayashi, Y. Aghakhani, and F. Dubeau, "Generalized epileptic discharges show thalamocortical activation and suspension of the default state of the brain," Proceedings of the National Academy of Sciences of the United States of America, vol. 102, no. 42, pp. 15236 15240, 2005.
[28] W. Liao, Z. Zhang, Z. Pan et al., "Altered functional connectivity and small-world in mesial temporal lobe epilepsy," PLoS ONE, vol. 5, no. 1, Article ID e8525, 2010.

[29] F. R. Pereira, A. Alessio, M. S. Sercheli et al., "Asymmetrical hippocampal connectivity in mesial temporal lobe epilepsy: evidence from resting state fMRI," BMC Neuroscience, vol. 11, article 66, 2010.

[30] A. B. Waites, R. S. Briellmann, M. M. Saling, D. F. Abbott, and G. D. Jackson, "Functional connectivity networks are disrupted in left temporal lobe epilepsy," Annals of Neurology, vol. 59, no. 2, pp. 335-343, 2006.

[31] A. E. Cavanna and F. Monaco, "Brain mechanisms of altered conscious states during epileptic seizures," Nature Reviews Neurology, vol. 5, no. 5, pp. 267-276, 2009.

[32] F. Bartolomei, F. Wendling, J. Régis, M. Gavaret, M. Guye, and P. Chauvel, "Pre-ictal synchronicity in limbic networks of mesial temporal lobe epilepsy," Epilepsy Research, vol. 61, no. 1-3, pp. 89-104, 2004.

[33] G. Bettus, F. Wendling, M. Guye et al., "Enhanced EEG functional connectivity in mesial temporal lobe epilepsy," Epilepsy Research, vol. 81, no. 1, pp. 58-68, 2008.

[34] J. Gotman and V. Levtova, "Amygdala-hippocampus relationships in temporal lobe seizures: a phase-coherence study," Epilepsy Research, vol. 25, no. 1, pp. 51-57, 1996.

[35] M. Guye, J. Régis, M. Tamura et al., "The role of corticothalamic coupling in human temporal lobe epilepsy," Brain, vol. 129, no. 7, pp. 1917-1928, 2006.

[36] H. Laufs, K. Hamandi, A. Salek-Haddadi, A. K. Kleinschmidt, J. S. Duncan, and L. Lemieux, "Temporal lobe interictal epileptic discharges affect cerebral activity in "default mode" brain regions," Human Brain Mapping, vol. 28, no. 10, pp. 1023-1032, 2007.

[37] M. Boly, C. Phillips, L. Tshibanda et al., "Intrinsic brain activity in altered states of consciousness: how conscious is the default mode of brain function?" Annals of the New York Academy of Sciences, vol. 1129, pp. 119-129, 2008.

[38] M. Boly, L. Tshibanda, A. Vanhaudenhuyse et al., "Functional connectivity in the default network during resting state is preserved in a vegetative but not in a brain dead patient," Human Brain Mapping, vol. 30, no. 8, pp. 2393-2400, 2009.

[39] M. Greicius, "Resting-state functional connectivity in neuropsychiatric disorders," Current Opinion in Neurology, vol. 21, no. 4, pp. 424-430, 2008.

[40] S. Laureys, M. Boly, and P. Maquet, "Tracking the recovery of consciousness from coma," Journal of Clinical Investigation, vol. 116, no. 7, pp. 1823-1825, 2006.

[41] A. Salek-Haddadi, K. J. Friston, L. Lemieux, and D. R. Fish, "Studying spontaneous EEG activity with fMRI," Brain Research Reviews, vol. 43, no. 1, pp. 110-133, 2003.

[42] L. Tshibanda, A. Vanhaudenhuyse, M. Boly et al., "Neuroimaging after coma," Neuroradiology, vol. 52, no. 1, pp. 1524, 2010.

[43] A. Vanhaudenhuyse, Q. Noirhomme, L. J. Tshibanda et al., "Default network connectivity reflects the level of consciousness in non-communicative brain-damaged patients," Brain, vol. 133, no. 1, pp. 161-171, 2010.

[44] P. J. Allen, O. Josephs, and R. Turner, "A method for removing imaging artifact from continuous EEG recorded during functional MRI," NeuroImage, vol. 12, no. 2, pp. 230-239, 2000.

[45] J. Gotman, J. R. Ives, P. Gloor et al., "Changes in interictal EEG spiking and seizure occurrence in humans," Epilepsia, vol. 23, pp. 432-433, 1982.

[46] J. Martinerie, C. Adam, M. le van Quyen et al., "Epileptic seizures can be anticipated by non-linear analysis," Nature Medicine, vol. 4, no. 10, pp. 1173-1176, 1998. 
[47] K. Kobayashi, C. J. James, T. Nakahori, T. Akiyama, and J. Gotman, "Isolation of epileptiform discharges from unaveraged EEG by independent component analysis," Clinical Neurophysiology, vol. 110, no. 10, pp. 1755-1763, 1999.

[48] T. P. Jung, S. Makeig, M. Westerfield, J. Townsend, E. Courchesne, and T. J. Sejnowski, "Analysis and visualization of single-trial event-related potentials," Human Brain Mapping, vol. 14, no. 3, pp. 166-185, 2001.

[49] F. Moeller, P. LeVan, and J. Gotman, "Independent component analysis (ICA) of generalized spike wave discharges in fMRI: comparison with general linear model-based EEGfMRI," Human Brain Mapping, vol. 32, no. 2, pp. 209-217, 2011.

[50] R. Rodionov, F. de Martino, H. Laufs et al., "Independent component analysis of interictal fMRI in focal epilepsy: comparison with general linear model-based EEG-correlated fMRI," NeuroImage, vol. 38, no. 3, pp. 488-500, 2007.

[51] G. Srivastava, S. Crottaz-Herbette, K. M. Lau, G. H. Glover, and V. Menon, "ICA-based procedures for removing ballistocardiogram artifacts from EEG data acquired in the MRI scanner," NeuroImage, vol. 24, no. 1, pp. 50-60, 2005.

[52] S. M. Smith, P. T. Fox, K. L. Miller et al., "Correspondence of the brain's functional architecture during activation and rest," Proceedings of the National Academy of Sciences of the United States of America, vol. 106, no. 31, pp. 13040-13045, 2009.

[53] H. Laufs and J. S. Duncan, "Electroencephalography/functional MRI in human epilepsy: what it currently can and cannot do," Current Opinion in Neurology, vol. 20, no. 4, pp. 417-423, 2007.

[54] M. Zijlmans, G. Huiskamp, M. Hersevoort, J. H. Seppenwoolde, A. C. van Huffelen, and F. S. Leijten, "EEG-fMRI in the preoperative work-up for epilepsy surgery," Brain, vol. 130, no. 9, pp. 2343-2353, 2007.

[55] J. Jacobs, P. LeVan, F. Moeller et al., "Hemodynamic changes preceding the interictal EEG spike in patients with focal epilepsy investigated using simultaneous EEG-fMRI," NeuroImage, vol. 45, no. 4, pp. 1220-1231, 2009.

[56] P. Federico, D. F. Abbott, R. S. Briellmann, A. S. Harvey, and G. D. Jackson, "Functional MRI of the pre-ictal state," Brain, vol. 128, no. 8, pp. 1811-1817, 2005.

[57] F. Grouiller, R. C. Thornton, K. Groening et al., "With or without spikes: localization of focal epileptic activity by simultaneous electroencephalography and functional magnetic resonance imaging," Brain, vol. 134, no. 10, pp. 28672886, 2011.

[58] D. King and S. Spencer, "Invasive electroencephalography in mesial temporal lobe epilepsy," Journal of Clinical Neurophysiology, vol. 12, no. 1, pp. 32-45, 1995.

[59] J. X. Tao, A. Ray, S. Hawes-Ebersole, and J. S. Ebersole, "Intracranial EEG substrates of scalp EEG interictal spikes," Epilepsia, vol. 46, no. 5, pp. 669-676, 2005.

[60] C. E. Châtillon, R. Zelmann, A. Bortel, M. Avoli, and J. Gotman, "Contact size does not affect high frequency oscillation detection in intracerebral EEG recordings in a rat epilepsy model," Clinical Neurophysiology, vol. 122, no. 9, pp. 1701-1705, 2011.

[61] J. D. Jirsch, E. Urrestarazu, P. LeVan, A. Olivier, F. Dubeau, and J. Gotman, "High-frequency oscillations during human focal seizures," Brain, vol. 129, no. 6, pp. 1593-1608, 2006.

[62] E. Urrestarazu, R. Chander, F. Dubeau, and J. Gotman, "Interictal high-frequency oscillations $(10-500 \mathrm{~Hz})$ in the intracerebral EEG of epileptic patients," Brain, vol. 130, no. 9, pp. 2354-2366, 2007.
[63] J. Jacobs, P. LeVan, R. Chander, J. Hall, F. Dubeau, and J. Gotman, "Interictal high-frequency oscillations $(80-500 \mathrm{~Hz})$ are an indicator of seizure onset areas independent of spikes in the human epileptic brain," Epilepsia, vol. 49, no. 11, pp. 1893-1907, 2008.

[64] R. RamachandranNair, A. Ochi, K. Imai et al., "Epileptic spasms in older pediatric patients: MEG and ictal high-frequency oscillations suggest focal-onset seizures in a subset of epileptic spasms," Epilepsy Research, vol. 78, no. 2-3, pp. 216224, 2008.

[65] A. Bragin, C. L. Wilson, J. Almajano, I. Mody, and J. Engel Jr., "High-frequency oscillations after status epilepticus: epileptogenesis and seizure genesis," Epilepsia, vol. 45, no. 9, pp. 1017-1023, 2004.

[66] E. Kobayashi, A. P. Bagshaw, C. G. Bénar et al., "Temporal and extratemporal BOLD responses to temporal lobe interictal spikes," Epilepsia, vol. 47, no. 2, pp. 343-354, 2006.

[67] F. Musso, J. Brinkmeyer, A. Mobascher, T. Warbrick, and G. Winterer, "Spontaneous brain activity and EEG microstates. A novel EEG/fMRI analysis approach to explore resting-state networks," NeuroImage, vol. 52, no. 4, pp. 1149-1161, 2010.

[68] A. P. Bagshaw, Y. Aghakhani, C. G. Bénar et al., "EEG-fMRI of focal epileptic spikes: analysis with multiple haemodynamic functions and comparison with gadolinium-enhanced MR angiograms," Human Brain Mapping, vol. 22, no. 3, pp. 179192, 2004.

[69] C. G. Bénar, D. W. Gross, Y. Wang et al., "The BOLD response to interictal epileptiform discharges," NeuroImage, vol. 17, no. 3, pp. 1182-1192, 2002.

[70] L. Lemieux, H. Laufs, D. Carmichael, J. S. Paul, M. C. Walker, and J. S. Duncan, "Noncanonical spike-related BOLD responses in focal epilepsy," Human Brain Mapping, vol. 29, no. 3, pp. 329-345, 2008.

[71] S. M. Mirsattari, Z. Wang, J. R. Ives et al., "Linear aspects of transformation from interictal epileptic discharges to BOLD fMRI signals in an animal model of occipital epilepsy," NeuroImage, vol. 30, no. 4, pp. 1133-1148, 2006.

[72] V. L. Morgan, J. C. Gore, and B. Abou-Khalil, "Functional epileptic network in left mesial temporal lobe epilepsy detected using resting fMRI," Epilepsy Research, vol. 88, no. 2-3, pp. 168-178, 2010.

[73] J. R. Sato, C. Rondinoni, M. Sturzbecher, D. B. de Araujo, and E. Amaro Jr., "From EEG to BOLD: brain mapping and estimating transfer functions in simultaneous EEG-fMRI acquisitions," NeuroImage, vol. 50, no. 4, pp. 1416-1426, 2010.

[74] M. J. McKeown, "Detection of consistently task-related activations in fMRI data with hybrid independent component analysis," NeuroImage, vol. 11, no. 1, pp. 24-35, 2000.

[75] Y. Aghakhani, A. P. Bagshaw, C. G. Bénar et al., "fMRI activation during spike and wave discharges in idiopathic generalized epilepsy," Brain, vol. 127, no. 5, pp. 1127-1144, 2004.

[76] G. Bettus, E. Guedj, F. Joyeux et al., "Decreased basal fMRI functional connectivity in epileptogenic networks and contralateral compensatory mechanisms," Human Brain Mapping, vol. 30, no. 5, pp. 1580-1591, 2009.

[77] H. Laufs, U. Lengler, K. Hamandi, A. Kleinschmidt, and K. Krakow, "Linking generalized spike-and-wave discharges and resting state brain activity by using EEG/fMRI in a patient with absence seizures," Epilepsia, vol. 47, no. 2, pp. 444-448, 2006.

[78] J. E. Motelow and H. Blumenfeld, "Functional neuroimaging of spike-wave seizures," Methods in Molecular Biology, vol. 489, pp. 189-209, 2009. 
[79] J. R. Tenney, T. Q. Duong, J. A. King, R. Ludwig, and C. F. Ferris, "Corticothalamic modulation during absence seizures in rats: a functional MRI assessment," Epilepsia, vol. 44, no. 9, pp. 1133-1140, 2003.

[80] H. Blumenfeld, M. Westerveld, R. B. Ostroff et al., "Selective frontal, parietal, and temporal networks in generalized seizures," NeuroImage, vol. 19, no. 4, pp. 1556-1566, 2003.

[81] H. Blumenfeld, K. A. McNally, S. D. Vanderhill et al., "Positive and negative network correlations in temporal lobe epilepsy," Cerebral Cortex, vol. 14, no. 8, pp. 892-902, 2004.

[82] D. J. Englot, A. M. Mishra, P. K. Mansuripur, P. Herman, F. Hyder, and H. Blumenfeld, "Remote effects of focal hippocampal seizures on the rat neocortex," Journal of Neuroscience, vol. 28, no. 36, pp. 9066-9081, 2008.

[83] W. T. Blume, "Current trends in electroencephalography," Current Opinion in Neurology, vol. 14, no. 2, pp. 193-197, 2001.

[84] M. Caporro, Z. Haneef, H. J. Yeh et al., "Functional MRI of sleep spindles and K-complexes," Clinical Neurophysiology, vol. 123, no. 2, pp. 303-309, 2012.

[85] H. Laufs, A. Kleinschmidt, A. Beyerle et al., "EEG-correlated fMRI of human alpha activity," NeuroImage, vol. 19, no. 4, pp. 1463-1476, 2003.

[86] N. K. Logothetis, J. Pauls, M. Augath, T. Trinath, and A. Oeltermann, "Neurophysiological investigation of the basis of the fMRI signal," Nature, vol. 412, no. 6843, pp. 150-157, 2001.

[87] F. A. Gibbs, W. G. Lennox, and E. L. Gibbs, "Cerebral blood flow preceding and accompanying epileptic seizures in man," Archives of Neurology and Psychiatry, vol. 32, pp. 257-272, 1934.

[88] Y. Nir, R. Mukamel, I. Dinstein et al. et al., "Interhemispheric correlations of slow spontaneous neuronal fluctuations revealed in human sensory cortex," Nature Neuroscience, vol. 11, no. 9, pp. 1100-1108, 2008.

[89] J. C. de Munck, S. I. Gonçalves, R. Mammoliti, R. M. Heethaar, and F. H. Lopes da Silva, "Interactions between different EEG frequency bands and their effect on alphafMRI correlations," NeuroImage, vol. 47, no. 1, pp. 69-76, 2009.

[90] B. J. He and M. E. Raichle, "The fMRI signal, slow cortical potential and consciousness," Trends in Cognitive Sciences, vol. 13, no. 7, pp. 302-309, 2009.

[91] H. Lu, Y. Zuo, H. Gu et al., "Synchronized delta oscillations correlate with the resting-state functional MRI signal," Proceedings of the National Academy of Sciences of the United States of America, vol. 104, no. 46, pp. 18265-18269, 2007.

[92] H. Laufs, K. Krakow, P. Sterzer et al., "Electroencephalographic signatures of attentional and cognitive default modes in spontaneous brain activity fluctuations at rest," Proceedings of the National Academy of Sciences of the United States of America, vol. 100, no. 19, pp. 11053-11058, 2003.

[93] L. Tyvaert, P. LeVan, C. Grova, F. Dubeau, and J. Gotman, "Effects of fluctuating physiological rhythms during prolonged EEG-fMRI studies," Clinical Neurophysiology, vol. 119 , no. 12, pp. 2762-2774, 2008.

[94] J. Britz, D. van de Ville, and C. M. Michel, "BOLD correlates of EEG topography reveal rapid resting-state network dynamics," NeuroImage, vol. 52, no. 4, pp. 1162-1170, 2010.

[95] S. S. Spencer, A. T. Berg, B. G. Vickrey et al., "Initial outcomes in the Multicenter Study of Epilepsy Surgery," Neurology, vol. 61, no. 12, pp. 1680-1685, 2003.

[96] N. B. Danielson, J. N. Guo, and H. Blumenfeld, "The default mode network and altered consciousness in epilepsy," Behavioural Neurology, vol. 24, no. 1, pp. 55-65, 2011.
[97] A. D. Norden and H. Blumenfeld, "The role of subcortical structures in human epilepsy," Epilepsy and Behavior, vol. 3, no. 3, pp. 219-231, 2002.

[98] R. L. Buckner, J. Sepulcre, T. Talukdar et al., "Cortical hubs revealed by intrinsic functional connectivity: mapping, assessment of stability, and relation to Alzheimer's disease," Journal of Neuroscience, vol. 29, no. 6, pp. 1860-1873, 2009.

[99] M. E. Raichle and A. Z. Snyder, "A default mode of brain function: a brief history of an evolving idea," NeuroImage, vol. 37, no. 4, pp. 1083-1090, 2007.

[100] B. Hahn, T. J. Ross, and E. A. Stein, "Cingulate activation increases dynamically with response speed under stimulus unpredictability," Cerebral Cortex, vol. 17, no. 7, pp. 1664-1671, 2007.

[101] M. D. Greicius, V. Kiviniemi, O. Tervonen et al., "Persistent default-mode network connectivity during light sedation," Human Brain Mapping, vol. 29, no. 7, pp. 839-847, 2008.

[102] S. G. Horovitz, A. R. Braun, W. S. Carr et al., "Decoupling of the brain's default mode network during deep sleep," Proceedings of the National Academy of Sciences of the United States of America, vol. 106, no. 27, pp. 11376-11381, 2009.

[103] F. Cauda, B. M. Micon, K. Sacco et al., "Disrupted intrinsic functional connectivity in the vegetative state," Journal of Neurology, Neurosurgery and Psychiatry, vol. 80, no. 4, pp. 429-431, 2009.

[104] S. Laureys, C. Lemaire, P. Maquet, C. Phillips, and G. Franck, "Cerebral metabolism during vegetative state and after recovery to consciousness," Journal of Neurology Neurosurgery and Psychiatry, vol. 67, no. 1, p. 121, 1999.

[105] S. Laureys, M. E. Faymonville, A. Luxen, M. Lamy, G. Franck, and P. Maquet, "Restoration of thalamocortical connectivity after recovery from persistent vegetative state," Lancet, vol. 355, no. 9217, pp. 1790-1791, 2000.

[106] P. Hagmann, L. Cammoun, X. Gigandet et al., "Mapping the structural core of human cerebral cortex," PLoS Biology, vol. 6, no. 7, article e159, 2008.

[107] B. J. Baars, "Global workspace theory of consciousness: toward a cognitive neuroscience of human experience," Progress in Brain Research, vol. 150, pp. 45-53, 2005.

[108] M. Arthuis, L. Valton, J. Rgis et al., "Impaired consciousness during temporal lobe seizures is related to increased longdistance corticalsubcortical synchronization," Brain, vol. 132, no. 8, pp. 2091-2101, 2009.

[109] F. Bartolomei and L. Naccache, "The global workspace (GW) theory of consciousness and epilepsy," Behavioural Neurology, vol. 24, no. 1, pp. 67-74, 2011.

[110] A. P. Bagshaw and A. E. Cavanna, "Brain mechanisms of altered consciousness in focal seizures," Behavioural Neurology, vol. 24, no. 1, pp. 35-41, 2011.

[111] T. Bayne, "The presence of consciousness in absence seizures," Behavioural Neurology, vol. 24, no. 1, pp. 47-53, 2011.

[112] A. E. Cavanna, "Epilepsy and disorders of consciousness," Behavioural Neurology, vol. 24, no. 1, p. 1, 2011.

[113] A. E. Cavanna and F. Ali, "Epilepsy: the quintessential pathology of consciousness," Behavioural Neurology, vol. 24, no. 1, pp. 3-10, 2011.

[114] M. Johanson, K. Valli, and A. Revonsuo, "How to assess ictal consciousness?" Behavioural Neurology, vol. 24, no. 1, pp. 1120, 2011.

[115] S. Seri, D. Brazzo, N. J. Thai, and A. Cerquiglini, "Brain mechanisms of altered consciousness in generalised seizures," Behavioural Neurology, vol. 24, no. 1, pp. 43-46, 2011.

[116] C. Koch, "The SCP is not specific enough to represent conscious content," Trends in Cognitive Sciences, vol. 13, no. 9, p. 367, 2009. 


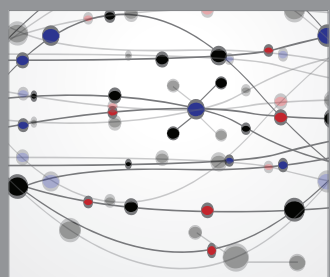

The Scientific World Journal
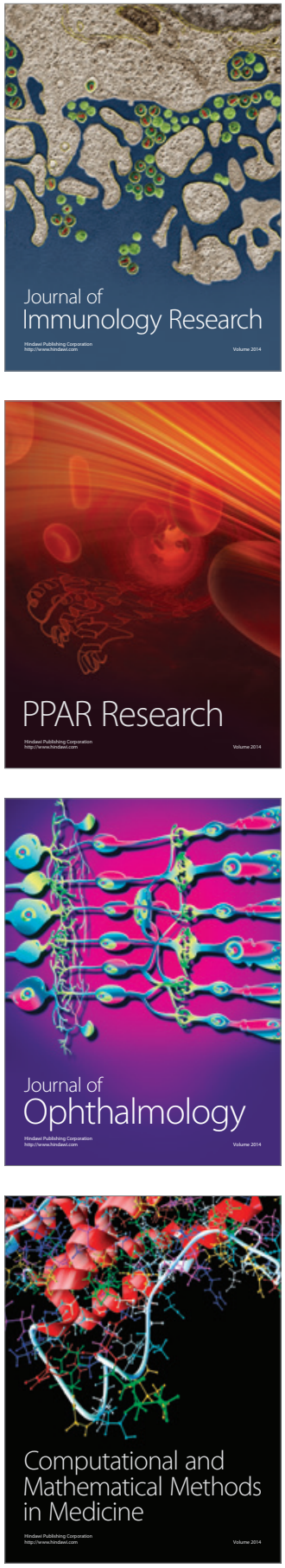

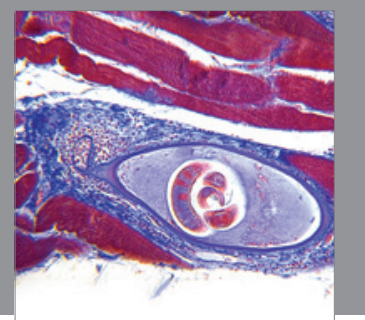

Gastroenterology

Research and Practice
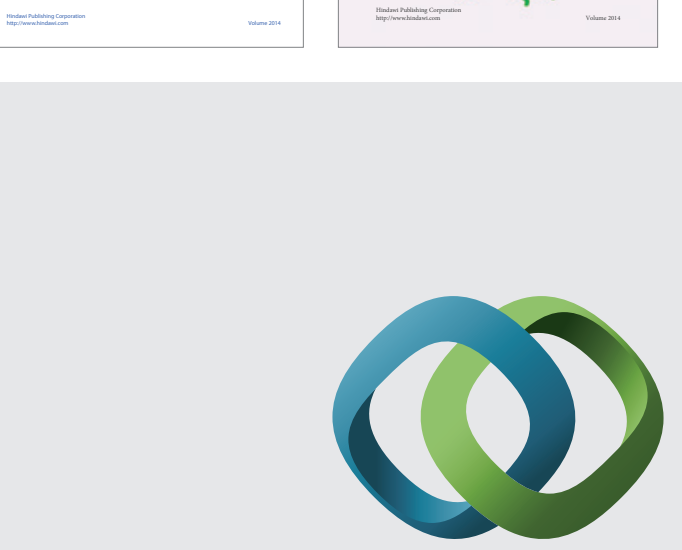

\section{Hindawi}

Submit your manuscripts at

http://www.hindawi.com
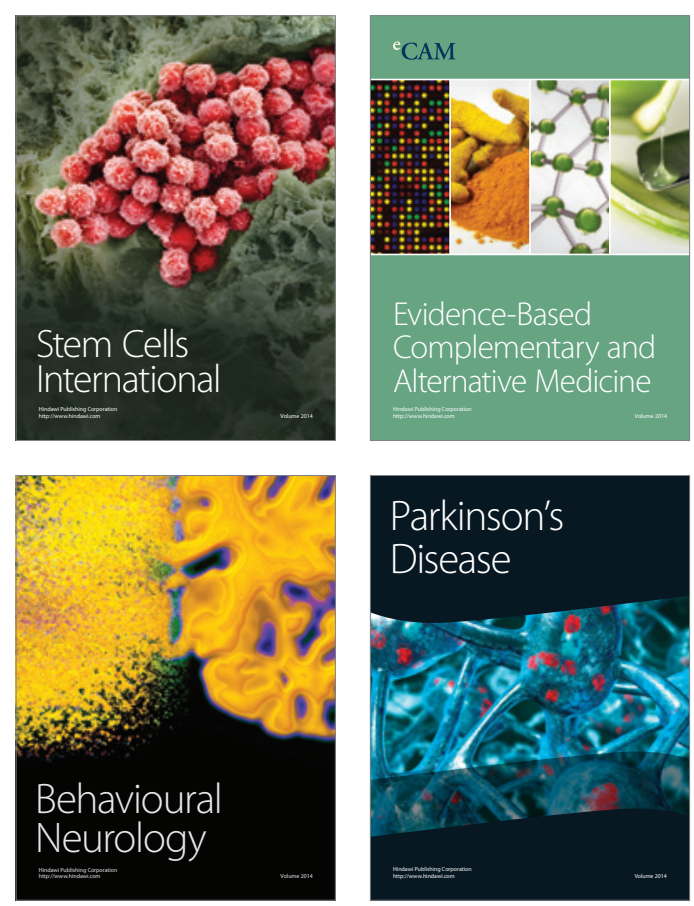

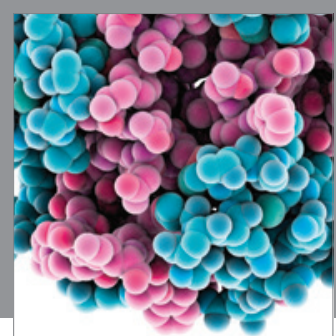

Journal of
Diabetes Research

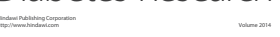

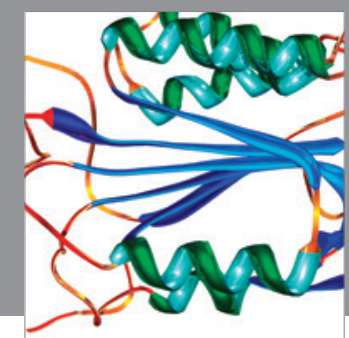

Disease Markers
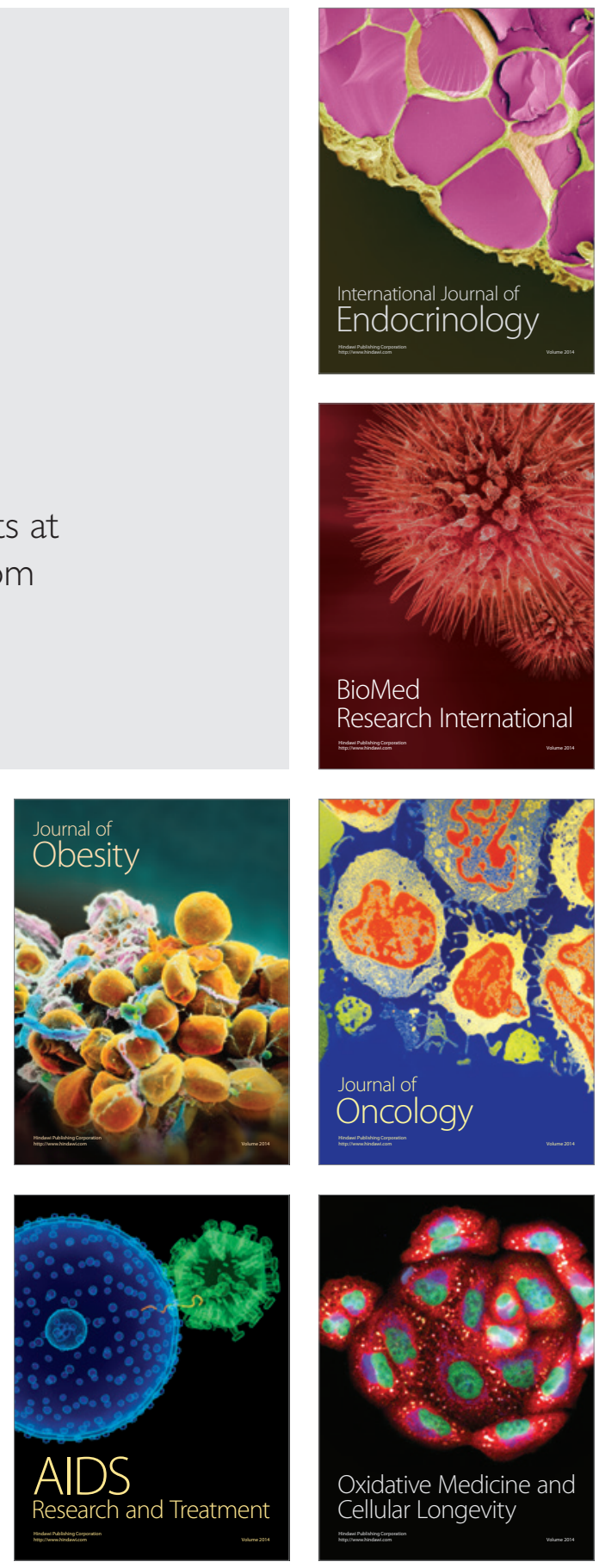